\title{
Network analysis of skin tumor progression identifies a rewired genetic architecture affecting inflammation and tumor susceptibility
}

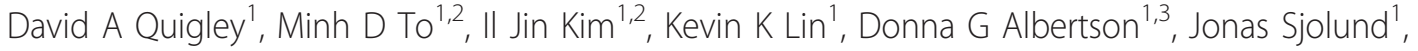
Jesús Pérez-Losada ${ }^{4}$, Allan Balmain ${ }^{1 *}$

\begin{abstract}
Background: Germline polymorphisms can influence gene expression networks in normal mammalian tissues and can affect disease susceptibility. We and others have shown that analysis of this genetic architecture can identify single genes and whole pathways that influence complex traits, including inflammation and cancer susceptibility. Whether germline variants affect gene expression in tumors that have undergone somatic alterations, and the extent to which these variants influence tumor progression, is unknown.
\end{abstract}

Results: Using an integrated linkage and genomic analysis of a mouse model of skin cancer that produces both benign tumors and malignant carcinomas, we document major changes in germline control of gene expression during skin tumor development resulting from cell selection, somatic genetic events, and changes in the tumor microenvironment. The number of significant expression quantitative trait loci (eQTL) is progressively reduced in benign and malignant skin tumors when compared to normal skin. However, novel tumor-specific eQTL are detected for several genes associated with tumor susceptibility, including IL18 (I/18), Granzyme E (Gzme), Sprouty homolog 2 (Spry2), and Mitogen-activated protein kinase kinase 4 (Map2k4).

Conclusions: We conclude that the genetic architecture is substantially altered in tumors, and that eQTL analysis of tumors can identify host factors that influence the tumor microenvironment, mitogen-activated protein (MAP) kinase signaling, and cancer susceptibility.

\section{Background}

Common genetic variants have been shown to affect many complex traits, including cancer susceptibility [1]. However, factors responsible for most of the expected heritable risk of cancer development have not yet been identified. Finding these alleles and isolating the causal polymorphisms is challenging because the heritable component of susceptibility is influenced by many alleles exerting modest effects that may be pleiotropic, epistatic, or context-dependent $[2,3]$. Mouse models of cancer using inbred strains of a defined genetic background do not recapitulate the genetic heterogeneity inherent in human populations. However, genetically heterogeneous mouse crosses permit analysis of the combinatorial

\footnotetext{
* Correspondence: abalmain@cc.ucsf.edu

${ }^{1}$ Helen Diller Family Comprehensive Cancer Center, University of California

San Francisco, 1450 Third St, San Francisco, CA 94158, USA

Full list of author information is available at the end of the article
}

effects of host genetic background and somatic events during tumor evolution, and these crosses have been used to identify polymorphisms that influence tumor susceptibility and progression [4-7]. Analysis of the genetic architecture of gene expression in normal skin from a Mus spretus/Mus musculus backcross ([SPRET/Ei X FVB/N] X FVB/N, hereafter FVBBX) identified expression quantitative trait loci (eQTL) that influence both structural and functional phenotypes, including hair follicle development, inflammation and tumor susceptibility [8]. A systematic analysis of germline influence on gene expression in benign and malignant skin tumors could identify novel alleles that influence tumorigenesis but are undetectable by analysis of normal tissue. Here we demonstrate that somatic alterations during tumor progression reduce the detectable influence of germline polymorphisms, but alleles that are not relevant in normal tissue are found to influence innate immune 
responses to skin tumors and are associated with tumor susceptibility.

\section{Results}

Germline control of gene expression is altered in tumors Skin tumors were induced on a cohort of 71 FVBBX mice by treatment of dorsal back skin with dimethyl benzanthracene (DMBA) and tetradecanoyl-phorbol acetate (TPA) (see experimental design in Figure S1 of Additional file 1). This treatment induced multiple benign papillomas as well as malignant carcinomas. Gene expression analysis was performed on mRNA extracted from 68 of these papillomas: two papillomas from each of 31 FVBBX mice and a single papilloma from six additional FVBBX mice. Gene expression and DNA copy number analysis was performed on 60 carcinomas that developed on these animals. A second cohort of 28 FVBBX animals (the 'confirmation' cohort) was subsequently generated and treated with the same carcinogenesis protocol as the first set of mice in order to confirm gene expression and eQTL results from the discovery cohort.

Germline polymorphisms have been shown to influence gene expression in tissues from model organisms and humans [8-13], but it is not clear how this influence is altered during tumor progression. If the germline plays no significant role in tumor gene expression, we would expect papilloma gene expression profiles from the same host to cluster near each other only by chance. Hierarchical clustering of gene expression profiles from papillomas demonstrated that tumors from the same mouse are most similar to each other in 19 of the 31 papilloma pairs (Figure 1a). The highly significant similarity of gene expression from same-host papillomas suggested that germline polymorphisms affect constitutive levels of gene expression in benign tumors $(P<$ 0.00001 by permutation; see Materials and methods). The contribution of genetic background to the benign and malignant tumor gene expression profiles was quantified by eQTL analysis. Our previous study of normal skin from the same animals identified almost 8,000 candidate $\mathrm{eQTL}$ at $\leq 10 \%$ false discovery rate (FDR). We identified 3,408 candidate eQTL in the 68 papillomas and 912 candidate eQTL in the 60 carcinomas significant at $\leq 10 \%$ FDR (Figure 1b; carcinoma eQTL listed in Table S1 in Additional file 1). At $\leq 5 \%$ FDR we identified 2,175 and 674 candidate eQTL in papillomas and carcinomas, respectively; increasing statistical stringency reduced the number of candidate eQTL but did not change the subsequent results qualitatively, and we report eQTL significant at the 10\% FDR level.

The striking reduction in eQTL detected in tumors, particularly in malignant carcinomas, prompted us to investigate reasons why fewer genes are significantly influenced by germline polymorphisms in carcinomas than in normal skin. Of the 7,414 genes with significant eQTL in skin, only 237 are not expressed in tumors, so complete absence of gene expression explains only about $3 \%$ of the decrease. EQTLs affecting genes that did not undergo drastic changes (more than two standard deviations from the mean fold-change) in their expression levels were more likely to be conserved between skin and carcinomas $(P<7.4 \mathrm{e}-06$, Fisher exact test). Conserved eQTL had significantly stronger statistical significance in normal skin than non-conserved eQTL $(P<1 \mathrm{e}-16$, Wilcoxon signed rank test). In normal skin we identified eQTL acting in cis (where the locus is physically proximal to the gene it affects) and in trans (where the locus is distant from or on another chromosome from the gene it affects) with approximately equal frequencies. The most statistically significant eQTL in skin acted overwhelmingly in cis. The cis/trans proportion detected in tails was $0.8 / 1$, while in papillomas it was approximately $1.5 / 1$, and in carcinomas it was approximately 5.75/1 (exact counts are listed in Table S2 in Additional file 1). We conclude that only very strong eQTL effects carry through from normal skin to affect the malignant carcinomas, and weaker transacting effects are rarely conserved.

\section{Somatic events alter the genetic architecture of gene expression in tumors}

Changes in the wiring of signaling pathways through epigenetic or genetic alterations may alter the influence of germline polymorphisms on gene expression in transformed cells. We used array comparative genomic hybridization $(\mathrm{aCGH})$ analysis to quantify alterations in tumor DNA copy number. Tumors showed widespread genomic instability (Figure 2a). The most frequent target of large-scale amplification in FVBBX carcinomas was distal chromosome 7 , which showed copy number gains in $45 \%$ (27 of 60 ) of carcinomas. Chromosome seven had a markedly smaller percentage of eQTL conserved between skin and carcinomas $(2.2 \%)$ than other autosomal chromosomes (mean $10 \%$, range $2.2 \%$ to $-15 \%$; Figure $2 \mathrm{~b}$ ). We identified a significant correlation between amplification of the most distal probe on chromosome 7 and fold-change increases of several genes located near the probe, including Ccnd1 (encoding Cyclin D1; $P=3.0 \mathrm{e}-6$, mean 10.5-fold up-regulation; Figure 2c). Cyclin D1 amplification or overexpression is an early event in numerous human tumors, and targeted over-expression of Ccnd1 drives several mouse models of carcinogenesis [14-16]. Although Ccnd1 had a significant cis-eQTL in skin (uncorrected $P=0.0001$, permutation $P=0.009, q<0.015)$, this cis-eQTL was not detected in papillomas or carcinomas.

DMBA induces a characteristic activating mutation in Hras1 [17], which is also located on distal chromosome 
(a)

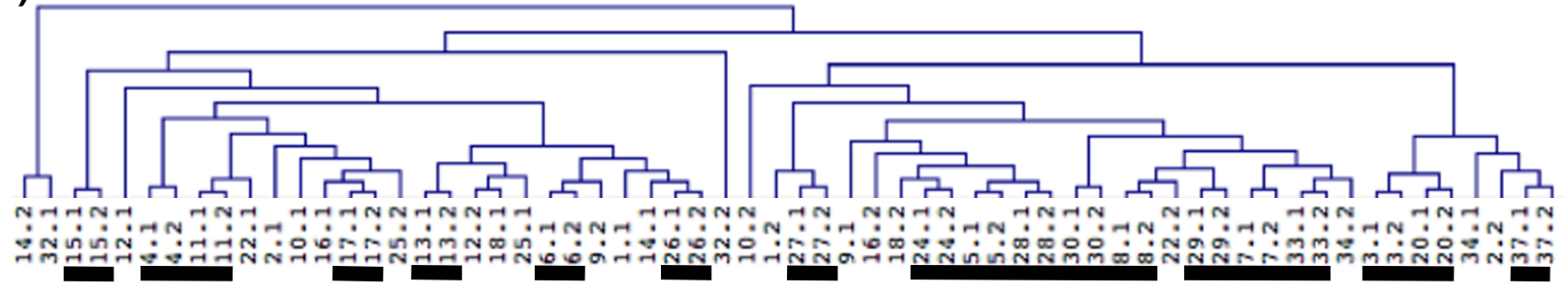

(b)

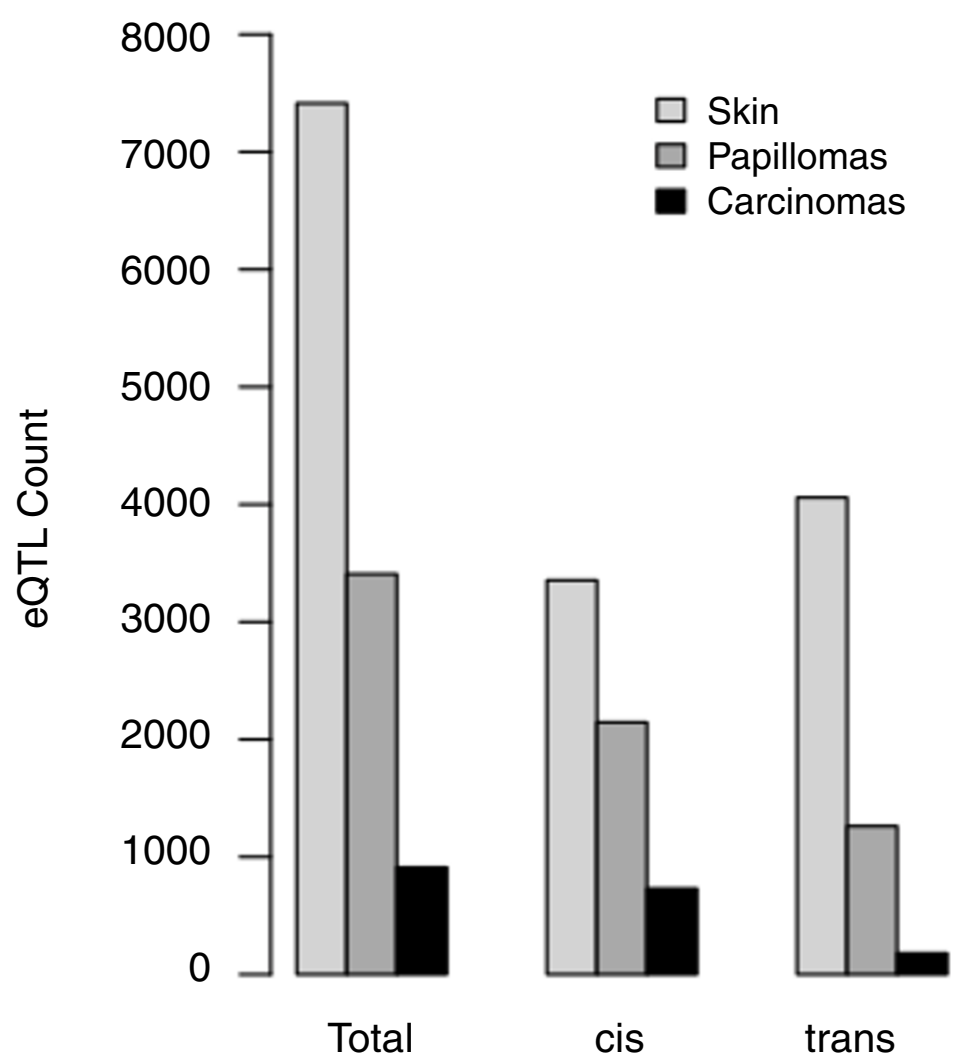

Figure 1 The influence of germline polymorphisms on gene expression is present but reduced in tumors. (a) Hierarchical clustering of total gene expression from papilloma pairs indicates that germline polymorphisms continue to exert a major effect on gene expression at the benign tumor stage. Bars indicate when both papillomas in a pair are most similar to each other. (b) Counts of total, cis-, and trans- eQTL in skin, papillomas, and carcinomas, showing that overall germline control of gene expression is strongly reduced, particularly for trans-eQTL, in malignant carcinomas.

7 in the mouse. Hras1 also had a significant cis-eQTL in skin (uncorrected $P=8.7 \mathrm{e}-5$, permutation $P=0.013, q<$ $0.02)$ that was not detected in papillomas or carcinomas. Changes in Hras 1 mutant gene copy number and/or loss of the normal wild-type allele play a role in tumor progression, and trisomy of chromosome 7 is a common early event in both papillomas and carcinomas, leading to increased copy number of the mutant Hras1 allele $[18,19]$. We conclude that gene copy number alterations on distal chromosome 7 have disrupted the normal genetic control of expression of these target genes.
Genomic networks are rewired during tumorigenesis

Changes in gene expression networks in tumors can result from macroscopic alterations in cellular composition during transformation, or from rewiring of signaling pathways. Coordinated alterations in gene expression from normal to tumor can be visualized as a 'progression network' by combining correlation and differential expression analysis (see Materials and methods; genes used to build this network and fold-change values are listed in Table S3 in Additional file 1). This method identifies functionally related gene sets with significantly 


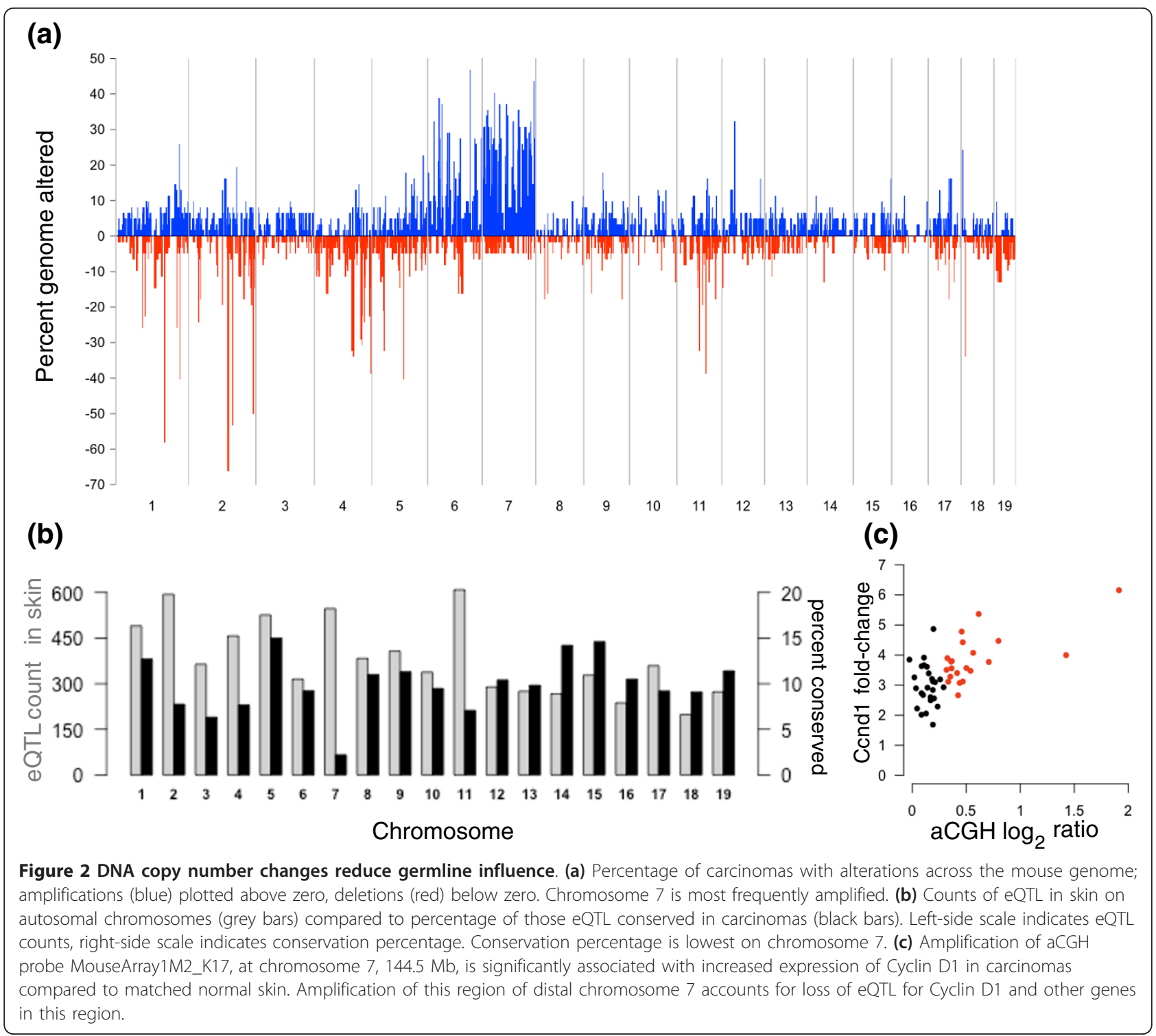

correlated changes in expression between two states. The global network constructed in this way is shown in Figure 3 and demonstrates that pathways linked to mitosis, stress responses, and IL1-mediated signaling are seen as distinct network motifs that are up-regulated in carcinomas. Carcinomas result from clonal expansion of initiated epidermal cells, and this is reflected in the down-regulation of motifs related to epithelial barrier, striated muscle, and hair follicles.

We previously identified a hair follicle network in normal skin genetically linked to the G-protein coupled receptor gene $\operatorname{Lgr} 5$, known to mark hair follicle stem cells $[8,20]$. Papillomas do not produce hair follicles, although they continue to express hair follicle keratins (Figure 4a; Figure S2 in Additional file 1). Although Lgr5 is significantly expressed in papillomas and carcinomas, it is not under the control of a cis-eQTL in tumors, and also is not linked genetically to the hair follicle correlation network. A papilloma-specific eQTL network including hair follicle keratins and keratin-associated proteins was detected with a shared locus of control on distal chromosome six (Figure 4b), a locus that was not significantly associated with these genes in normal tissue. The G-protein coupled receptor family member Gprc5d was the only cis-eQTL in the new network (raw $P=$ 5.4e-4, permutation $P=0.02, q=0.02$; linkage map plotted in Figure S3 in Additional file 1). Intriguingly, overexpression of Gprc5d promotes hair keratin gene expression, and Gprc5d is expressed in whn (hairless) nude mice [21], compatible with a role that would only be revealed when normal hair follicle control has been disrupted. These data suggest that the hair follicle stem 


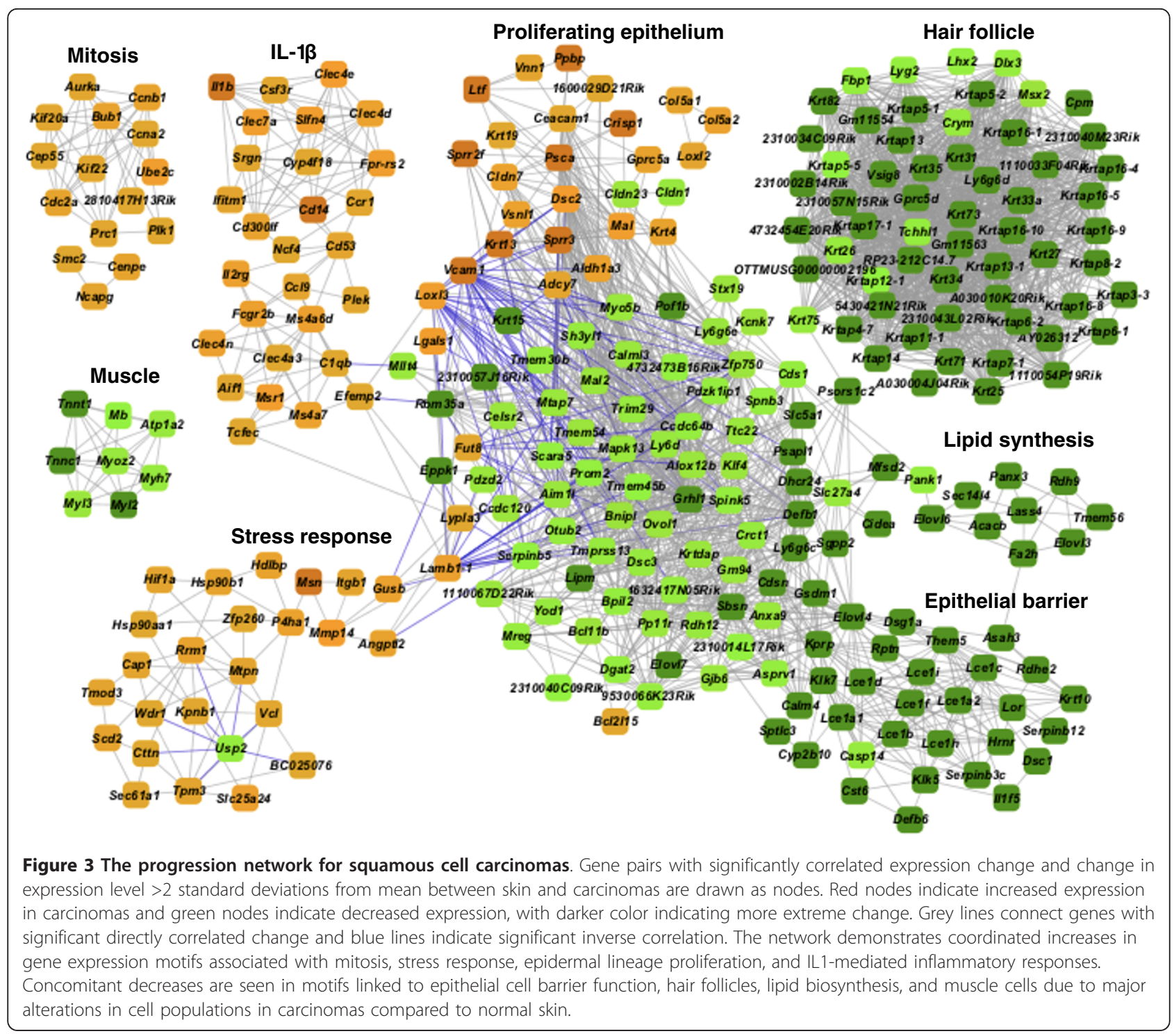

cell network is significantly rewired during skin tumor development, but the possible role of $\operatorname{Lgr} 5$ as a marker of tumor initiating cells remains to be determined. We conclude that gene copy number changes, somatic mutations, and alterations in tissue composition in papillomas and carcinomas account for the loss of the Ccnd1, Hras1, and Lgr5 eQTL and likely are responsible for the loss of many other eQTL seen in normal skin.

\section{Tumor-specific eQTL are associated with susceptibility}

Of the 912 transcripts with significant eQTL in carcinoma, 210 did not have a significant eQTL in normal skin (carcinoma eQTL are listed in Table S1 in Additional file 1). Of the 210 eQTL detected only in carcinomas, in 45 cases the transcript was expressed only in carcinomas and not in normal tissue. This may be due to activation of signaling pathways not expressed in normal skin, or by infiltration of transformed epithelium by cell populations from the microenvironment not normally resident in the skin, particularly cells of the innate and adaptive immune systems. Loci that affect the expression of transcripts in tumors but not normal skin may affect tumor susceptibility, but these eQTL would not be evident from analysis of normal tissue. To identify genes with tumor-specific eQTL that were associated with susceptibility, we identified genes that were significantly differentially expressed when contrasting papillomas from resistant and susceptible animals (FDR $\leq 5 \%$ ). Genes were considered of interest if they had expression in papillomas significantly associated with susceptibility and also had a tumor-specific eQTL.

Twenty-nine genes met these criteria (listed in Table 1). Of these genes, the serine protease Granzyme E (Gzme) showed the largest induction in papillomas from 

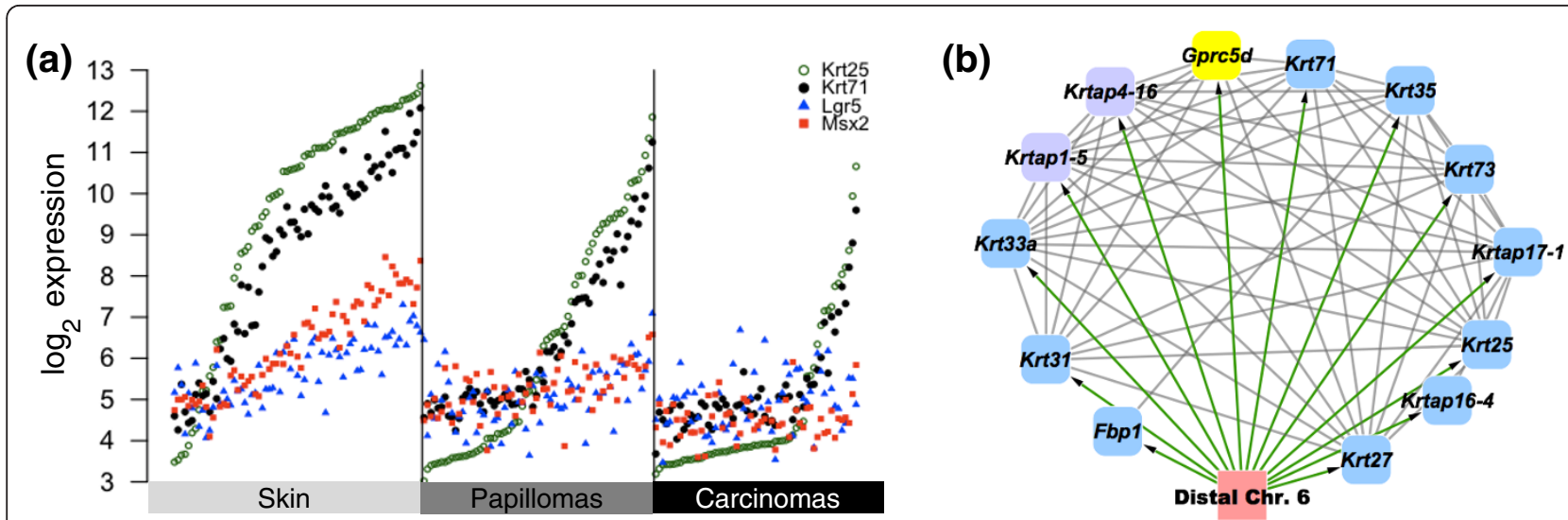

Figure 4 Re-wiring of the Lgr5 hair follicle eQTL network. (a) Gene expression levels of Krt71, Krt25, Msx2, and Lgr5 in skin, papillomas, and carcinomas, showing that while Lgr5 is significantly correlated with Msx2 and Krt71 in normal skin, this association is lost during tumor progression. (b) A new eQTL network for hair follicle keratins in papillomas where the locus affects Gprc5d (yellow node) in cis and other genes (blue nodes) in trans. Green lines indicate significant influence of eQTL locus on all genes in the network ( $\leq 10 \%$ FDR); grey lines indicate significant gene-gene correlation.

resistant mice. Gzme is expressed in granules released by cytotoxic $\mathrm{T}$ lymphocytes and together with perforin can destroy pathogen-infected or transformed cells [22,23]. Gzme was expressed at background levels in normal FVBBX skin, but at a range of detectable levels in papillomas and carcinomas (Figure 5a). The tumor-specific ciseQTL for Gzme peaked at chromosome 14, $51 \mathrm{Mb}$ in papillomas and carcinomas (raw $P=6.6 \mathrm{e}-7$, permutation $P<0.001, q<0.001$; Figure $5 \mathrm{~b}$ ). Mice heterozygous at the eQTL locus (that is, with Gzme alleles inherited from both FVB/N and SPRET/Ei) had higher expression of Gzme in papillomas and carcinomas than mice homozygous for $\mathrm{FVB} / \mathrm{N}$ at this allele. Although (as previously reported [8]) classical QTL analysis of papilloma counts for these FVBBX mice did not identify a locus significant after multiple test correction, the strongest linkage was to markers on chromosome 14, peaking at $62 \mathrm{Mb}$ (linkage map plotted in Figure S4 in Additional file 1). The SPRET/Ei allele was protective at this locus, in agreement with the direction of the Gzme eQTL and susceptibility results. We conclude that Gzme is a strong candidate modifier of papilloma susceptibility based on genetic control of gene expression in tumor tissue, higher levels of expression in papillomas from resistant mice carrying the SPRET/Ei allele, and the documented biological activity of granzymes in killing of potential tumor cells.

Higher expression of several other genes was associated with resistance, including Sprouty homolog two (Spry2), a negative regulator of Ras/mitogen-activated protein kinase (MAPK) signaling (raw $P=7.6 \mathrm{e}-8$, permutation $P<0.01, q=0.03)$. Spry 2 was also expressed at very low levels in normal skin, but was expressed at elevated levels in tumors. The DMBA/TPA model of carcinogenesis is driven by oncogenic signaling through the Ras pathway, and it is plausible that mice with higher constitutive levels of Spry 2 expression in tumors would show greater resistance to tumorigenesis.

Some genes associated with susceptibility are expressed in normal skin but are only under germline control in tumors. The IL1 family member IL18 (Il18) was expressed in skin and tumor samples, but only in carcinomas did $I l 18$ have a strong cis-eQTL, with higher expression in papillomas from susceptible animals and when a SPRET/Ei allele was present (raw $P=2.6 \mathrm{e}-8$, permutation $P<0.001, q=0.001$ ). Higher levels of the kinase Map2k4 (also called Mek4 or Mkk4) are also associated with increased susceptibility, and this gene is under germline control only in tumors (raw $P=3.5 \mathrm{e}-5$, permutation $P=0.005, q=0.014$ ). A recent report has shown that FVB mice with a skin-specific knockout of Map $2 k 4$ are resistant to the DMBA/TPA tumorigenesis protocol, consistent with our eQTL analysis [24].

\section{Perturbation from normal expression is controlled primarily in trans}

The tumor eQTL analysis described above was based on steady state levels of all transcripts detected in tumors. The availability of matched normal skin and tumor tissue enabled us to ask whether the degree of perturbation of transcript levels in the tumors, as opposed to their steady state levels, is under germline control. We performed an eQTL analysis on the gene expression changes when comparing the same probe in matched normal skin and carcinomas. Including only probes that were expressed above background in both skin and carcinomas and that did not have a significant eQTL in tail or carcinoma analysis based on steady-state levels, we identified 55 significant eQTL. In contrast to carcinoma 
Table 1 Genes with novel eQTL in tumors that are also associated with susceptibility

\begin{tabular}{|c|c|c|c|c|c|c|c|c|c|}
\hline Symbol & Probe & Chr. & $\mathrm{Mb}$ & Fold change & SAM $q$-value & Higher in & Higher genotype & eQTL chr. & eQTL Mb \\
\hline Gzme & 1421227_at & 14 & 56.7 & -16.67 & $<0.01$ & Resist. & Het. & 14 & 41 \\
\hline Gzme & 1450171_x_at & 14 & 56.7 & -7.69 & $<0.01$ & Resist. & Het. & 14 & 41 \\
\hline Mnda & 1452349_x_at & 1 & 175.8 & -2.94 & 4.31 & Resist. & Het. & 1 & 169 \\
\hline 2310005E10Rik & 1453173_at & 6 & 34.3 & -2.27 & 3.02 & Resist. & Het. & 6 & 32 \\
\hline$D d x 6$ & 1439122_at & 9 & 44.4 & -1.82 & 4.31 & Resist. & Hom. & 9 & 34 \\
\hline Spry2 & 1421656_at & 14 & 106.3 & -1.39 & 3.02 & Resist. & Het. & 14 & 94 \\
\hline Kctd3 & 1436811_at & 1 & 190.8 & 1.16 & 4.31 & Susc. & Hom. & 1 & 187 \\
\hline Map2k4 & 1451982_at & 11 & 65.5 & 1.16 & 1.38 & Susc. & Hom. & 11 & 101 \\
\hline Ssr1 & 1441327_a_at & 13 & 38.1 & 1.18 & 3.02 & Susc. & Hom. & 10 & 106 \\
\hline Ndst2 & 1417931_at & 14 & 21.5 & 1.21 & 3.02 & Susc. & Hom. & 14 & 19 \\
\hline Ppih & 1429832_at & 4 & 119.0 & 1.23 & 2.02 & Susc. & Hom. & 5 & 44 \\
\hline 1810063B07Rik & 1427905_at & 14 & 20.9 & 1.23 & 1.38 & Susc. & Hom. & 14 & 23 \\
\hline Psme3 & 1418078_at & 11 & 101.2 & 1.25 & 3.02 & Susc. & Hom. & 4 & 75 \\
\hline Tardbp & 1436318_at & 4 & 148.0 & 1.25 & 2.02 & Susc. & Hom. & 1 & 187 \\
\hline BC003266 & 1449189_at & 4 & 126.9 & 1.25 & $<0.01$ & Susc. & Hom. & 4 & 121 \\
\hline Acbd6 & 1452601_a_at & 1 & 157.4 & 1.26 & 2.02 & Susc. & Het. & 9 & 116 \\
\hline 2810457l06Rik & 1436805_at & 9 & 40.8 & 1.26 & 0.93 & Susc. & Het. & 9 & 34 \\
\hline Dhdds & 1450654_a_at & 4 & 133.5 & 1.26 & $<0.01$ & Susc. & Hom. & 4 & 141 \\
\hline Nrd1 & 1424391_at & 4 & 108.7 & 1.28 & 0.93 & Susc. & Hom. & 10 & 118 \\
\hline Nme6 & 1448574_at & 9 & 109.7 & 1.3 & 0.93 & Susc. & Het. & 9 & 102 \\
\hline Sept8 & 1426802_at & 11 & 53.3 & 1.35 & 2.02 & Susc. & Hom. & 4 & 106 \\
\hline Hyls 1 & 1431315_at & 9 & 35.4 & 1.38 & 1.38 & Susc. & Het. & 9 & 34 \\
\hline Pus3 & 1418491_a_at & 9 & 35.4 & 1.42 & 0.93 & Susc. & Het. & 9 & 34 \\
\hline C230096C10Rik & 1436709_at & 4 & 138.9 & 1.43 & 1.38 & Susc. & Hom. & 4 & 141 \\
\hline Cregl & 1415947_at & 1 & 167.7 & 1.46 & 2.02 & Susc. & Hom. & 1 & 160 \\
\hline Asah3l & 1451355_at & 4 & 86.5 & 1.51 & 0.33 & Susc. & Hom. & 13 & 1 \\
\hline Rdh11 & 1449209_a_at & 12 & 80.3 & 1.57 & 3.02 & Susc. & Het. & 6 & 32 \\
\hline Mtap2 & 1434194_at & 1 & 66.2 & 1.81 & 1.38 & Susc. & Hom. & 10 & 102 \\
\hline Ts/p & 1450004_at & 18 & 33.0 & 2.34 & 2.02 & Susc. & Het. & 5 & 138 \\
\hline 1118 & 1417932_at & 9 & 50.4 & 2.34 & $<0.01$ & Susc. & Het. & 9 & 34 \\
\hline
\end{tabular}

Genes that satisfy two conditions: rewired or novel eQTL in tumors compared to normal skin and significant differential expression in papillomas when tumors from resistant and susceptible mice are compared. 'Chr.' and ' $\mathrm{Mb}$ ' indicate gene location; 'Fold change' indicates differential expression between resistant and susceptible; 'SAM q-value' indicates differential expression significance; 'Higher in' indicates whether the gene was higher in resistant (Resist.) or susceptible (Susc.) animals; 'Higher genotype' indicates whether heterozygous (Het.) or homozygous (Hom.) alleles were associated with higher expression; 'eQTL chr.' and 'eQTL Mb' indicate peak eQTL linkage.

\section{(a)}

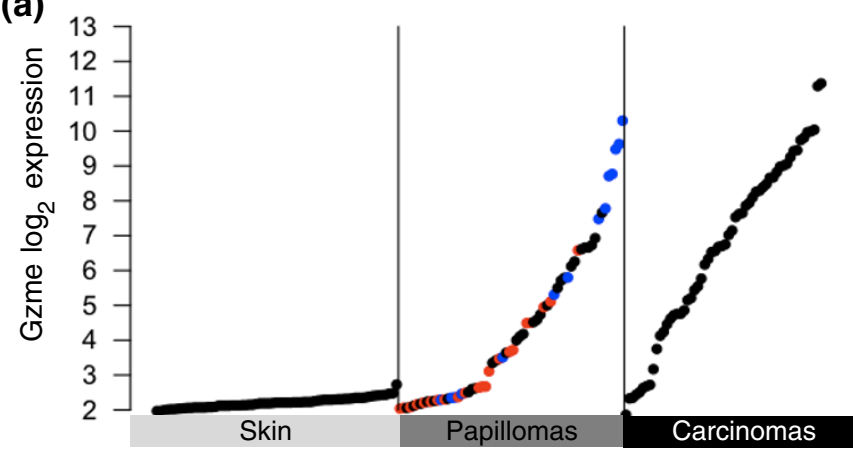

(b)

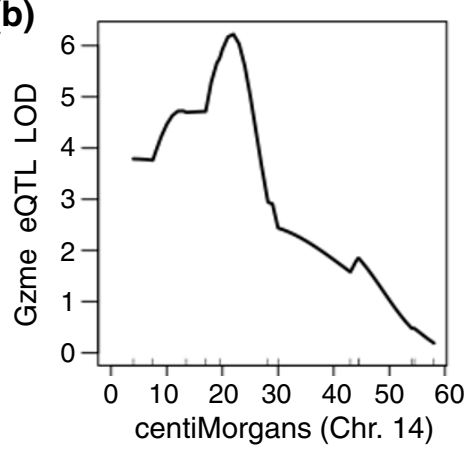

Figure 5 Granzyme E alleles are associated with su sceptibility. (a) $\log _{2}$ expression of Gzme in skin, papillomas, and carcinomas. Gzme mRNA is not detected in normal skin, and its level of expression is highest in papillomas from mice that are relatively resistant to papilloma development. Papillomas from resistant animals are plotted as blue circles, susceptible animals as red circles. (b) Expression of Gzme in papillomas and carcinomas is under germline genetic control. LOD plot for Gzme carcinoma eQTL significance on chromosome 14. 
eQTL, which acted almost exclusively in cis, $80 \%$ of these novel 'perturbation eQTL' acted in trans (44 of 55; listed in Table S4 in Additional file 1). A recent report investigating gene expression changes in human cell lines in response to ionizing radiation demonstrated that loci associated with response overwhelmingly acted in trans [25]. It is possible, therefore, that major perturbations of gene expression as a result of DNA damage or tumor development are controlled indirectly through the influence of trans-acting regulatory factors (for example, transcription factors) rather than through widespread influence on transcription levels of individual genes.

\section{Confirmation of tumor eQTL}

Of the 912 transcripts with significant eQTL in the discovery carcinoma eQTL data set, 560 were significant in the confirmation cohort at a 5\% FDR. The number of samples in the confirmation cohort was relatively small $(N=28)$, and it is possible that more predicted eQTL would have been confirmed with a more highly powered study. These eQTL were mostly cis-eQTL and included the eQTL affecting Gzme and Il18 expression (Figure S5 in Additional file 1; replication results listed in Table S1 in Additional file 1).

\section{Discussion}

The past few years have witnessed an explosion in genome-wide association studies of cancer susceptibility in human populations. While these studies have revealed many new genetic variants that influence cancer risk, each variant is predicted to have a very small effect on susceptibility, and most heritable factors influencing risk remain to be discovered [26]. Some risk is conferred by rare variants with large effects, such as the BRCA1/ $B R C A 2$ mutations that increase breast cancer susceptibility. Rare variations cannot be detected by genomewide association studies, which analyze only common (typically $>5 \%$ minor allele frequency) alleles. Epistatic interactions between common alleles may also contribute to cancer risk. The latter model is supported by studies of mouse models of cancer susceptibility, which have demonstrated that common alleles interact in a complex fashion to influence risk [27]. However, even in mouse models that combine defined inbred strains with dramatically different tumor susceptibilities under wellcontrolled environmental conditions, classical mapping studies have not identified the majority of the risk factors [28].

The realization that cancer susceptibility is an emergent property of the combinatorial effects of many genes necessitated the development of more complex networkbased approaches that integrate classical genetics with gene expression analysis in normal and transformed tissues. We have previously used a systems genetics approach to analyze how gene expression networks in normal whole skin vary between animals that are susceptible or resistant to skin papilloma development. This approach led to identification of pathways controlling mitosis, inflammation and tissue remodeling in normal skin that affect individual susceptibility [8]. In the present study we have focused on analysis of the rewiring of these normal gene expression networks during development of benign and malignant tumors from the same heterogeneous population of inter-specific backcross mice.

Our data illuminate the dynamic changes in cell populations, both tumor-derived and host-derived, that accompany the evolution of solid tumors. Genomic networks in squamous cell carcinomas are profoundly deregulated compared to normal epithelium and benign papillomas, reflecting major changes in gross tissue organization and signaling. Allelic variation continues to influence tumor gene expression, although this influence is reduced by the somatic alterations accompanying progression. The strongest reduction in tumors is seen in eQTL that act in trans, possibly due to genomic instability leading to alterations in transcription factor-mediated control of gene expression and the tissue-specific nature of trans-eQTL. eQTL under the control of cis-acting elements in general have stronger effects than trans-eQTL, and they may be more robust in the face of somatic genetic changes because the causal variant affects the gene directly. A recent study compared eQTL detected in hematopoietic cells at four stages of differentiation and demonstrated that many eQTL are unique to each state, and trans-eQTL are less likely to be conserved between differentiation states than cis-eQTL [29]. Trans-eQTL were detected in all four states.

We have also identified 'perturbation eQTL', which measure the degree to which changes in levels of gene expression between normal and transformed states are under genetic control. These eQTL reflect genetic control of the changes that occur in response to exogenous damage. In contrast to the steady state eQTL that are mainly cis-acting, perturbation eQTL act primarily in trans, similar to a scenario recently described for human lymphoblastoid cells subjected to ionizing radiation [25]. The mechanistic basis for these observations remain to be determined by isolation and analysis of the trans-acting factors responsible for these effects.

Genetic and gene expression analyses of tumors reveals features that cannot be detected by analysis of normal tissues, such as the cis-eQTL controlling expression of Il18, Gzme, Map2k4, and Spry2 in tumors but not normal skin. Il18 has an important and complex role in inflammatory and immune responses; it has been reported to have both tumor-promoting and anti-tumor activities in different contexts [30]. It remains to be 
determined whether the gain of germline influence over Il18 expression reflects a change in cell populations or a modification in cell-autonomous signaling. The presence of a tumor-specific eQTL for $I l 18$ may reflect differences in the relative proportions of epithelial and inflammatory cells in the tumors, or may be due to rewiring of Il18 signaling during progression.

Unlike Il18, Gzme expression is not detectable in normal skin, and appears in papillomas and carcinomas concomitantly with the influx of innate immune cells. Mice with higher levels of Gzme within their papillomas were relatively resistant to papilloma development, in agreement with a protective role for Gzme, and possibly other granzymes within this gene cluster, in tumor development. In contrast, mice with high levels of $I l 18$ in their papillomas were most susceptible to tumor development. These data suggest that innate immune cell responses against tumors are stronger in animals that carry the SPRET/Ei allele at the Gzme locus, due to a polymorphism resulting in higher Gzme expression. This analysis also suggests opposing roles in tumor susceptibility for Map2k4 and Spry2, genes that exert opposite effects on mitogen-activated protein kinase (MAPK) signaling.

Tumor signaling can be rewired due to oncogenic mutations or loss of tumor suppressor genes, possibly revealing activity of a germline polymorphism that is not evident in normal tissue. The identification of susceptibility genes by a combination of genetic and gene expression analysis of tumors highlights the power of this approach to elucidate the genetic architecture of cancer susceptibility. A combination of genetic and gene expression analysis of human tumors will complement genetic association methods and may identify additional susceptibility factors that cannot be detected using classical methods.

\section{Materials and methods}

\section{Mouse models, gene expression, and aCGH}

FVBBX mice were generated and treated with DMBA/ TPA as described in [8]. Gene expression was measured with the Affymetrix Mouse Genome 4302.0 microarray, Affymetrix annotation release 30 . Microarray probesets where all 11 probes did not hybridize to an annotated Refseq gene were eliminated from analysis. Animals sensitive to papilloma tumorigenesis were defined as $>7$ papillomas after 20 weeks of treatment $(N=22)$, resistant as $<2$ papillomas at that time point $(N=11)$. The confirmation cohort of FVBBX mice was generated and treated by the same protocol, with genotypes and gene expression measured as described above. Genomic amplification/deletion was measured with a 2,504 probe aCGH system using $\log _{2} \pm 0.3$ cutoffs for amplification/deletion [31]. Percentage of the genome altered was calculated by dividing each chromosome into 1,000,000 equally spaced bins and calling each bin amplified or deleted depending on the status of the most physically proximal probe for which a measurement was available. Statistical analysis was performed with the R package [32].

\section{Statistical analysis of gene expression}

Permutation analysis of hierarchical clustering was performed by first calculating the distance matrix for sample gene expression using all present genes, counting cases where the closest papilloma to a given sample was from the same mouse $\left(\mathrm{N}_{\text {observed }}\right)$. We performed 10,000 permutations of the sample labels and calculated $\mathrm{N}_{\text {perm }}$ in the same manner, reporting the number of times $\mathrm{N}_{\text {perm }} \geq \mathrm{N}_{\text {observed }}$ divided by 10,000. Differential expression was analyzed with the Significance Analysis of Microarrays algorithm [33]. Correlation was defined as significant at the $5 \%$ alpha level using the experimentwise genome-wide error rate permutation method as described in [34]. To calculate the tumor progression network, skin and carcinoma microarrays were normalized together and genotype-matched skin expression was subtracted from tumor expression. Mean foldchange values were approximately normally distributed. Highly significant change for progression networks was defined as $>2$ standard deviations from the global mean change $(N=926)$. Significant correlation in fold-change was assessed at the $5 \%$ genome-wide level using the genome-wide error rate method as described in [34]. All significantly correlated pairs of probes with highly significant fold-change in expression and membership in a network clique of size 3 or greater were plotted. Correlation networks were drawn using Cytoscape [35]. Microarray results have been deposited in the Gene Expression Omnibus under accession number [GEO: GSE21264].

\section{eQTL analysis}

Pairs of papillomas from the same animal were combined for eQTL analysis using the mean expression for each probeset. eQTL were identified by linear regression as previously described [8]. Briefly, corrected eQTL $P$-values were calculated by storing the lowest observed $P$-value $p_{\text {minimal-obs }}$ across all $230 \mathrm{SNPs}$ and generating 1,000 shuffled genotypes, calculating $p_{\text {minimal-perm }}$ for each permutation, and reporting the rank of $p_{\text {minimal-obs }}$ in the sorted set of $p_{\text {minimal-perm }}$ divided by 1,000 . The distribution of corrected $P$-values was used as input to Storey's QVALUE software [36] to calculate FDR $q$-values. Candidate cis-eQTL were defined as loci within $30 \mathrm{Mb}$ of the gene they were predicted to affect (qualitatively similar results were obtained with windows of 20 and $40 \mathrm{Mb}$ ). Interval mapping was performed with R/QTL [37]. The 912 carcinoma eQTL from the 
discovery cohort were tested in the confirmation dataset by linear regression of the loci and gene expression values. The distribution of 912 confirmation $P$-values was used with QVALUE to calculate $q$-values for confirmation results.

\section{Additional material}

Additional file 1: Additional figures and tables. A schematic overview of the experiment, additional detailed figures supporting the eQTL analysis, a table listing eQTL detected in carcinomas, a table detailing cisand trans-eQTL counts, a table listing genes altered more than two standard deviations from the mean in carcinomas compared to matched normal skin, and a table listing perturbation eQTL identified.

\section{Abbreviations}

aCGH: array comparative genomic hybridization; DMBA: dimethyl benzanthracene; eQTL: expression quantitative trait locus/loci; FDR: false discovery rate; FVBBX: [SPRET/Ei X FVB/N] X FVB/N; IL: interleukin; TPA: tetradecanoyl-phorbol acetate.

\section{Acknowledgements}

This work was supported by the National Cancer Institute. AB acknowledges support from the Barbara Bass Bakar Chair of Cancer Genetics. MDT was supported in part by a Sandler Foundation postdoctoral research fellowship. JS was supported by the Swedish Research Council and the Tegger Foundation. KKL was supported by an NIH Kirschstein-NRSA postdoctoral research fellowship. JPL is partially supported by Carlos III (FIS)/FEDER, MICIIN/plan-E 2009, JCyL ('Biomedicina y Educación') and CSIC. The funders had no role in study design, data collection and analysis, decision to publish or preparation of the manuscript. We thank H Quigley, MH Barcellos-Hoff, RJ Akhurst and members of the Balmain lab for critical reading of the manuscript, $\mathrm{K}$ Jen for assistance with histology, and $\mathrm{JH}$ Mao and $\mathrm{H}$ Nagase for discussions in the early stages of this work.

\section{Author details}

${ }^{1}$ Helen Diller Family Comprehensive Cancer Center, University of California San Francisco, 1450 Third St, San Francisco, CA 94158, USA. ${ }^{2}$ Thoracic Oncology Program, Department of Surgery, University of California San Francisco, 1600 Divisadero St, San Francisco, CA 94143, USA. ${ }^{3}$ Department of Laboratory Medicine, University of California San Francisco, 1521 Parnassus Ave, Room C255, Box 0451, San Francisco, CA 94143, USA. ${ }^{4}$ Instituto de Biología Molecular y Celular del Cáncer, CSIC/Universidad de Salamanca, Campus M Unamuno s/n, 37007-Salamanca, Spain.

\section{Authors' contributions}

The study was conceived and supervised by AB. Bioinformatics analysis was performed by DAQ. RNA and DNA extraction was performed by $I K, K K L$, and MDT. JPL contributed mice and tumor samples and KKL performed array analysis for the confirmation study. Taqman assays were performed by JS. Array CGH data were provided by DGA. The paper was written by DAQ and $A B$.

Received: 16 October 2010 Revised: 2 December 2010

Accepted: 18 January 2011 Published: 18 January 2011

\section{References}

1. McCarthy MI, Abecasis GR, Cardon LR, Goldstein DB, Little J, loannidis JPA, Hirschhorn JN: Genome-wide association studies for complex traits: consensus, uncertainty and challenges. Nat Rev Genet 2008, 9:356-369.

2. Quigley D, Balmain A: Systems genetics analysis of cancer susceptibility: from mouse models to humans. Nat Rev Genet 2009, 10:651-657.

3. Flint J, Mackay TF: Genetic architecture of quantitative traits in mice, flies, and humans. Genome Res 2009, 19:723-733.
4. Nagase H, Bryson S, Cordell H, Kemp CJ, Fee F, Balmain A: Distinct genetic loci control development of benign and malignant skin tumours in mice. Nat Genet 1995, 10:424-429.

5. Ruivenkamp CA, van Wezel T, Zanon C, Stassen AP, Vlcek C, Csikos T, Klous AM, Tripodis N, Perrakis A, Boerrigter L, Groot PC, Lindeman J, Mooi WJ, Meijjer GA, Scholten G, Dauwerse H, Paces V, van Zandwijk N, van Ommen GJ, Demant P: Ptprj is a candidate for the mouse colon-cancer susceptibility locus Scc1 and is frequently deleted in human cancers. Nat Genet 2002, 31:295-300.

6. Ewart-Toland A, Briassouli P, de Koning JP, Mao JH, Yuan J, Chan F, MacCarthy-Morrogh L, Ponder BA, Nagase H, Burn J, Ball S, Almeida M, Linardopoulos S, Balmain A: Identification of Stk6/STK15 as a candidate low-penetrance tumor-susceptibility gene in mouse and human. Nat Genet 2003, 34:403-412.

7. Park YG, Zhao X, Lesueur F, Lowy DR, Lancaster M, Pharoah P, Qian X, Hunter KW: Sipa1 is a candidate for underlying the metastasis efficiency modifier locus Mtes1. Nat Genet 2005, 37:1055-1062.

8. Quigley D, To M, Pérez-Losada J, Pelorosso F, Mao J, Nagase H, Ginzinger D, Balmain A: Genetic architecture of mouse skin inflammation and tumour susceptibility. Nature 2009, 458:505-508.

9. Kristensen VN, Edvardsen H, Tsalenko A, Nordgard SH, Sørlie T, Sharan R, Vailaya A, Ben-Dor A, Lønning PE, Lien S, Omholt S, Syvänen AC, Yakhini Z, Børresen-Dale AL: Genetic variation in putative regulatory loci controlling gene expression in breast cancer. Proc Natl Acad Sci USA 2006, 103:7735-7740.

10. Chesler EJ, Lu L, Shou S, Qu Y, Gu J, Wang J, Hsu HC, Mountz JD, Baldwin NE, Langston MA, Threadgill DW, Manly KF, Williams RW: Complex trait analysis of gene expression uncovers polygenic and pleiotropic networks that modulate nervous system function. Nat Genet 2005 , 37:233-242.

11. Morley M, Molony CM, Weber TM, Devlin JL, Ewens KG, Spielman RS, Cheung VG: Genetic analysis of genome-wide variation in human gene expression. Nature 2004, 430:743-747

12. Brem RB, Yvert G, Clinton R, Kruglyak L: Genetic dissection of transcriptional regulation in budding yeast. Science 2002, 296:752-755.

13. Schadt EE, Monks SA, Drake TA, Lusis AJ, Che N, Colinayo V, Ruff TG, Milligan SB, Lamb JR, Cavet G, Linsley PS, Mao M, Stoughton RB, Friend SH: Genetics of gene expression surveyed in maize, mouse and man. Nature 2003, 422:297-302

14. Wang TC, Cardiff RD, Zukerberg L, Lees E, Arnold A, Schmidt EV: Mammary hyperplasia and carcinoma in MMTV-cyclin D1 transgenic mice. Nature 1994, 369:669-671.

15. Diehl JA: Cycling to cancer with cyclin D1. Cancer Biol Ther 2002, 1:226-231.

16. Opitz OG, Harada H, Suliman Y, Rhoades B, Sharpless NE, Kent R, Kopelovich L, Nakagawa H, Rustgi AK: A mouse model of human oralesophageal cancer. J Clin Invest 2002, 110:761-769.

17. Quintanilla M, Brown K, Ramsden M, Balmain A: Carcinogen-specific mutation and amplification of Ha-ras during mouse skin carcinogenesis. Nature 1986, 322:78-80.

18. Aldaz CM, Trono D, Larcher F, Slaga TJ, Conti CJ: Sequential trisomization of chromosomes 6 and 7 in mouse skin premalignant lesions. Mol Carcinog 1989, 2:22-26

19. Bremner $\mathrm{R}$, Balmain $\mathrm{A}$ : Genetic changes in skin tumor progression: correlation between presence of a mutant ras gene and loss of heterozygosity on mouse chromosome 7. Cell 1990, 61:407-417.

20. Jaks V, Barker $N$, Kasper $M$, van Es JH, Snippert HJ, Clevers H, Toftgård R: Lgr5 marks cycling, yet long-lived, hair follicle stem cells. Nat Genet 2008, 40:1291-1299.

21. Inoue $S$, Nambu T, Shimomura T: The RAIG family member, GPRC5D, is associated with hard-keratinized structures. J Invest Dermatol 2004, 122:565-573.

22. Jenne DE, Tschopp J: Granzymes, a family of serine proteases released from granules of cytolytic T lymphocytes upon T cell receptor stimulation. Immunol Rev 1988, 103:53-71.

23. Cullen SP, Brunet M, Martin SJ: Granzymes in cancer and immunity. Cell Death Differ 2010, 17:616-623.

24. Finegan $\mathrm{KG}$, Tournier $\mathrm{C}$ : The mitogen-activated protein kinase kinase 4 has a pro-oncogenic role in skin cancer. Cancer Res 2010, 70:5797-5806. 
25. Smirnov D, Morley M, Shin E, Spielman R, Cheung V: Genetic analysis of radiation-induced changes in human gene expression. Nature 2009, 459:587-591.

26. Manolio TA, Collins FS, Cox NJ, Goldstein DB, Hindorff LA, Hunter DJ, McCarthy MI, Ramos EM, Cardon LR, Chakravarti A, Cho JH, Guttmacher AE, Kong A, Kruglyak L, Mardis E, Rotimi CN, Slatkin M, Valle D, Whittemore AS, Boehnke M, Clark AG, Eichler EE, Gibson G, Haines JL, Mackay TF, McCarroll SA, Visscher PM: Finding the missing heritability of complex diseases. Nature 2009, 461:747-753.

27. Nagase $\mathrm{H}, \mathrm{MaO} J \mathrm{H}$, de Koning JP, Minami T, Balmain A: Epistatic interactions between skin tumor modifier loci in interspecific (spretus/ musculus) backcross mice. Cancer Res 2001, 61:1305-1308.

28. Mackay TFC, Stone EA, Ayroles JF: The genetics of quantitative traits: challenges and prospects. Nat Rev Genet 2009, 10:565-577.

29. Gerrits A, Li Y, Tesson BM, Bystrykh LV, Weersing E, Ausema A, Dontje B, Wang $X$, Breitling R, Jansen RC, de Haan G: Expression quantitative trait loci are highly sensitive to cellular differentiation state. PLoS Genet 2009, 5:e1000692.

30. Vidal-Vanaclocha F, Mendoza L, Telleria N, Salado C, Valcarcel M, Gallot N, Carrascal T, Egilegor E, Beaskoetxea J, Dinarello CA: Clinical and experimental approaches to the pathophysiology of interleukin-18 in cancer progression. Cancer Metastasis Rev 2006, 25:417-434.

31. Snijders AM, Nowak NJ, Huey B, Fridlyand J, Law S, Conroy J, Tokuyasu T, Demir K, Chiu R, Mao JH, Jain AN, Jones SJ, Balmain A, Pinkel D, Albertson DG: Mapping segmental and sequence variations among laboratory mice using BAC array CGH. Genome Res 2005, 15:302-311.

32. $R$ Core Development Team: $R$ : A language and environment for statistical computing Vienna, Austria: R Foundation for Statistical Computing; 2010.

33. Tusher VG, Tibshirani R, Chu G: Significance analysis of microarrays applied to the ionizing radiation response. Proc Natl Acad Sci USA 2001, 98:5116-5121.

34. Churchill GA, Doerge RW: Empirical threshold values for quantitative trait mapping. Genetics 1994, 138:963-971.

35. Shannon P, Markiel A, Ozier O, Baliga NS, Wang JT, Ramage D, Amin N, Schwikowski B, Ideker T: Cytoscape: a software environment for integrated models of biomolecular interaction networks. Genome Res 2003, 13:2498-2504.

36. Storey JD, Tibshirani R: Statistical significance for genomewide studies. Proc Natl Acad Sci USA 2003, 100:9440-9445.

37. Broman KW, Wu H, Sen S, Churchill GA: R/qtl: QTL mapping in experimental crosses. Bioinformatics 2003, 19:889-890.

38. Quigley D, To M, Pérez-Losada J, Pelorosso F, Mao J, Nagase H, Ginzinger D, Balmain A: Genetic architecture of mouse skin inflammation and tumour susceptibility. Nature 2009, 458:505-508.

39. Ciobanu DC, Lu L, Mozhui K, Wang X, Jagalur M, Morris JA, Taylor WL, Dietz K, Simon P, Williams RW: Detection, validation, and downstream analysis of allelic variation in gene expression. Genetics 2010, 184:119-128.

doi:10.1186/gb-2011-12-1-r5

Cite this article as: Quigley et al: Network analysis of skin tumor progression identifies a rewired genetic architecture affecting inflammation and tumor susceptibility. Genome Biology 2011 12:R5.

\section{Submit your next manuscript to BioMed Central and take full advantage of:}

- Convenient online submission

- Thorough peer review

- No space constraints or color figure charges

- Immediate publication on acceptance

- Inclusion in PubMed, CAS, Scopus and Google Scholar

- Research which is freely available for redistribution

Submit your manuscript at www.biomedcentral.com/submit
Biomed Central 